\title{
La educación científica infantil con perspectiva ecológica y social para la formación de ciudadanías participativas
}

\author{
María del Carmen Martínez-Silva ${ }^{1}$ \\ Gloria Elena Cruz-Sánchez ${ }^{1} \odot$ \\ Raquel Aparicio-Cid ${ }^{1}$ \\ ${ }^{1}$ Universidad Veracruzana (UV), México.
}

Resumen. La educación científica ha sido revalorada en este siglo por su potencial incidencia en la formación de una ciudadanía más dotada de mejores herramientas cognitivas para comprender el mundo, en base al acceso de una vasta información especializada prácticamente sobre cualquier campo. Sin embargo, estos elementos, por sí solos, resultan insuficientes para enfrentar los escenarios de incertidumbre impuestos por la crisis socioambiental. En este artículo se plantea una discusión teórica en torno al necesario giro pedagógico en la enseñanza de la ciencia desde los primeros años de vida, así como su complementariedad con la educación ambiental, como posibles alternativas para trascender las limitaciones de una educación en ciencia principalmente racionalista. Entre las principales conclusiones de esta discusión se propone la necesidad de integrar a las dimensiones ética, estética y política en la educación científica con personas en primera infancia, que contribuya a la formación de una ciudadanía comprometida con formas de vida socialmente más justas y sostenibles. Palabras clave: educación científica; educación ambiental; educación preescolar; ciudadanía.

A educação científica na educação infantil a partir de uma perspectiva ecológica e social para a formação de cidadãos participativos

Resumo. A educação científica foi reconsiderada neste século pelo seu impacto potencial na formação de uma cidadania dotada de melhores ferramentas cognitivas para compreender o mundo, com base no acesso a uma grande quantidade de informações especializadas em praticamente qualquer campo. No entanto, estes elementos por si só são insuficientes para enfrentar os cenários de incerteza impostos pela crise socioambiental. Este artigo apresenta uma discussão teórica sobre a necessidade de uma mudança pedagógica no ensino de ciências desde os primeiros anos de vida, bem como da sua complementaridade com a educação ambiental, como alternativas possíveis para transcender as limitações de uma educação científica predominantemente baseada no racionalismo. Entre as principais conclusões desta discussão está a necessidade de integrar as dimensões ética, estética e política na educação cientifica de crianças na primeira infância, contribuindo para a formação de cidadãos comprometidos com formas de vida socialmente mais justas e sustentáveis.

Palavras chave: educação científica; educação ambiental; educação infantil; cidadania.

\begin{abstract}
Children's scientific education with an ecological and social perspective for the formation of participatory citizenships

Abstract. Scientific education has been revalued in this century, for its potential impact on the formation of a citizenry endowed with better cognitive tools to understand the world, based on access to vast specialized information on practically any area. However, these elements, by themselves, are insufficient to face the uncertainty scenarios imposed by the socio-environmental crisis. This article presents a theoretical discussion around the necessary pedagogical turn in the teaching of science from the first years of life, as well as its complementarity with environmental education, as possible alternatives to transcend the limitations of a mainly rationalist science education. Among the main conclusions of this discussion is the need to integrate the ethical, aesthetic and political dimensions in scientific education with people in early childhood that contributes to the formation of a citizenry committed to more socially just and sustainable ways of life.
\end{abstract}

Keywords: science education, environmental education, preschool education, citizenry. 


\section{Introducción}

Los retos que plantean a la humanidad las grandes problemáticas socioambientales demandan una profunda transformación cultural (Ángel-Maya, 2003) en la que el conocimiento y la educación científica resulten esenciales. Sin embargo, por sí solos, los conocimientos de la ciencia no son suficientes para entender ni resolver los problemas cruciales de la sustentabilidad (Manifiesto por la vida, 2002), como tampoco basta la alfabetización científica para generar cambios significativos en la relación de las personas entre sí y con el ambiente (González-Gaudiano y Meira, 2020). La causa principal del distanciamiento entre la didáctica de las ciencias y la construcción de una sociedad sostenible subyace, según Torres (2010), en el arraigo de la ciencia al positivismo, lo que incide en las formas de concebir a la propia ciencia y la manera tradicionalista de enseñarla.

Con la intención de fortalecer la educación científica y tecnológica de cara a los desafíos de la sociedad actual y, recientemente, con mayor énfasis en educación infantil, diferentes organismos internacionales han generado numerosos informes y directrices en busca de impulsar la calidad en esta área de formación, como: los Objetivos para el Desarrollo Sostenible (ODS), propuestos por la Organización de las Naciones Unidas para la Educación, la Ciencia y la Cultura (UNESCO); la Organización para la Cooperación y el Desarrollo Económicos (OCDE), a través del Programa para la Evaluación Internacional de Alumnos (PISA, por sus siglas en inglés); y, el Laboratorio Latinoamericano para la Evaluación de la Calidad de la Educación de la UNESCO (LLECE), mediante la emisión de los Estudios Regionales Comparativos y Explicativos (ERCE) en la región latinoamericana, entre los más importantes. ${ }^{1}$

En el caso específico de México, como consecuencia de estas políticas educativas internacionales derivaron algunas acciones como el fortalecimiento de la investigación en educación superior a través del Consejo Nacional de Ciencia y la Tecnología (CONACYT), la aplicación de pruebas estandarizadas EXCALE en 2005 y PISA en 2006, y cambios curriculares con énfasis en la enseñanza de la ciencia para la educación básica en las reformas educativas desde 2009 hasta la última en 2017 (Ferra, Méndez y Castro, 2019). Concretamente, en el currículo de educación preescolar se establecieron estándares curriculares que definían los conocimientos, habilidades, actitudes y valores asociados a la ciencia que los niños debían aprender al concluir la educación preescolar (SEP, 2011). Lo anterior propició progresiones diferenciadas sobre las prácticas de enseñanza y aprendizaje en educación científica, como lo relatan investigaciones sobre formación docente en didáctica de las ciencias en diferentes países de habla hispana (cf. Porlán, Rivero y Martín del Pozo, 1997, 1998; Cañal, Travé y Pozuelos, 2011; Rivero, Martín del Pozo, Solís, Azcárate y Porlán, 2017; Morales y Colorado, 2019; Quintanilla, Orellana y Páez, 2020; Cruz-Guzmán, Puig y García, 2020).

Flores (2012) advierte que uno de los principales motivos del rezago en la investigación científica se encuentra enraizado en la educación básica, la cual se asocia con enfoques tradicionalistas centrados en la transmisión de conocimientos, lo que deriva en una concepción equívoca o limitada de la actividad científica y, en conse-

\footnotetext{
${ }^{1}$ Véanse los informes en: https://bit.ly/3iDjk5p, https://bit.ly/3uUbUzv, https://www.preal.online/ y https://bit.ly/3Dnulj7
} 
cuencia, de rechazo a la misma. Al respecto, Morales y Colorado (2019) coinciden en el reconocimiento de profundos retos en la formación docente inicial y el equipamiento de las aulas de ciencia en México.

En tal contexto, el objetivo de este ensayo teórico es discutir la visión prevaleciente de la educación científica asociada a la transmisión de contenidos conceptuales o de herramientas lógicas de pensamiento, como base para fundamentar la pertinencia de una educación eco-socio-científica en la formación temprana de ciudadanos comprometidos con una vida socialmente más justa y sostenible. Estos planteamientos emergen de una investigación en curso, que busca explicar el vínculo entre prácticas de educación científica y de educación ambiental en el currículo vivido en el nivel preescolar en México. La investigación estudia ¿cómo un colectivo docente diseña y pone en marcha un programa de educación científica vinculado a prácticas de educación ambiental para la comprensión del mundo?, a partir de proyectos de investigación escolar que emergen de preguntas e inquietudes de los niños preescolares.

La investigación requirió una exhaustiva búsqueda de nociones teóricas que contribuyan en la definición de un marco explicativo de partida, entendidas como estructuras discursivas que inciden en la definición de un objeto de estudio, su problematización y categorización para el análisis (Buenfil, 2006). En esta revisión teórica se trató de reconocer, en distintas vertientes del pensamiento educativo, ideas que faciliten la comprensión de la realidad, como lógicas de intelección, además de integrar una postura ética, política y onto-epistemológica sobre la construcción del conocimiento (Jiménez, 2006).

Bajo este orden de ideas, se definieron tres campos o áreas de conocimiento educativo para centrar la búsqueda de teorías generales y sus intersecciones: educación científica, educación ambiental y educación infantil. Para ello, se exploró de manera general la producción científica más reciente sobre estos temas en cuatro bases de datos (SciELO, Redalyc, Dialnet, Google académico) y el repositorio de la Universidad Veracruzana. A partir de ello se identificaron en las perspectivas contemporáneas y los fundamentos psicopedagógicos actuales, las teorías sustantivas útiles para la comprensión del objeto de estudio, con la intención de articular un entramado teórico bajo los paradigmas crítico e interpretativo que, bajo el supuesto de la relevancia de la educación científica en los primeros años de vida, respondiera las siguientes preguntas: ¿Por qué es importante la educación científica? ¿A qué se refiere? ¿Cuáles son los objetivos la educación científica infantil? ¿Cuáles son las limitaciones de la educación científica frente los escenarios actuales y cómo superarlas?

La información seleccionada bajo tales criterios se analizó a partir de seis categorías: fundamentos pedagógicos, dimensiones de formación, noción-relación con el medio ambiente, noción de ciencia, y sus principales alcances y limitaciones.

Como resultado de este proceso se integró un corpus teórico, desde el cual se exponen algunos de los argumentos centrales en torno al objeto de estudio, organizados en dos apartados. En el primero se discuten algunos elementos clave para contribuir a la formación ciudadana desde la educación científica en los escenarios actuales, como es el papel del maestro para potenciar la curiosidad infantil en los primeros años de vida -al considerarse la etapa infantil un momento clave para conocer, relacionarse y comprometerse con el ambiente; y en el segundo apartado se 
articula la complementariedad entre la educación científica y la educación ambiental, para lo cual se ponen en tensión convergencias y divergencias entre dichos campos. Para finalizar, se presentan las principales conclusiones derivadas de este ejercicio analítico-reflexivo.

\section{En busca de una educación científica que fortalezca la formación de identidades ciudadanas participativas}

Frente a la demanda de una mejor educación científica se identifica una serie de desafíos, entre los que se distingue el papel del maestro para aprovechar la curiosidad infantil, como un recurso clave en la manera de enseñar y aprender ciencia, la cual incide en la formación de una ciudadanía informada y participativa.

Para Quintanilla et al. (2020) y Cruz-Guzmán et al. (2020), la movilización de saberes para transitar hacia un modelo didáctico menos tradicionalista resulta un proceso complejo y multifactorial; y proponen considerar en el análisis de la formación docente para la enseñanza de la ciencia tanto a elementos curriculares (contenidos, materiales, recursos, espacio y organización), como el papel del docente y del alumno. Además de lo anterior, Ortiz y Cervantes (2015) distinguen a la concepción que el sujeto tiene sobre la ciencia como un elemento clave que determina la forma en que ésta se enseña. Para estos autores "la ciencia no es entendida como un conjunto de conocimientos, verdades absolutas o saberes ya establecidos que deben ser transmitidos, sino como una constante búsqueda de respuestas a las preguntas que los individuos se plantean sobre su realidad" (p. 12). Esta perspectiva enriquece la enseñanza que se hace de la ciencia por parte del docente, especialmente en la infancia.

De esta manera, dado que la ciencia es ante todo una manera de aproximarse a la comprensión de la realidad, resulta esencial incorporarla en los procesos educativos desde los primeros años de vida del ser humano, donde la formulación de preguntas es una constante, gracias a la curiosidad infantil, la cual es un rasgo fundamental en el desarrollo del pensamiento que impulsa el deseo por conocer (Dewey, 1989). En cuanto a la escuela, Tonucci (1997) la define como un laboratorio donde los niños exponen sus inquietudes, son escuchados y orientados en la búsqueda de respuestas para comprender el mundo y para transformarlo.

Entonces, si los niños son investigadores natos, cuya curiosidad es una fuente inagotable de impulsos cognitivos y afectivos para construir conocimientos, y la escuela es el escenario propicio para guiar a los infantes en el descubrimiento de su mundo, ¿cuál es el papel del docente y de la escuela en este desafío pedagógico? Una respuesta tentativa a esta pregunta es, en primera instancia, que el papel del maestro radica en generar las condiciones o ambientes de aprendizaje para la realización de investigaciones escolares que partan de la curiosidad infantil y que tomen como base la relatividad del conocimiento, rechazando la idea de verdades dogmáticas (Porlán et al., 1998).

Todo lo anterior permite asumir que la ciencia no puede ser enseñada como conocimiento construido, sino como una manera de comprender la realidad, de conocerla e interrogarla, a través de un proceso donde se integran emociones, apegos, pensamientos y principios éticos. El reto para la escuela y el docente es transitar de una pedagogía basada en respuestas a una pedagogía de preguntas, esto es, abandonar 
la trasnmisión de conocimientos, como verdades absolutas e incuestionables. Tonucci (2019) explica que, ante una pregunta infantil, el papel de los adultos alrededor es plantear más preguntas que ayuden al niño a desarrollar su pensamiento y con ello, brindarles la oportunidad de cuestionar y pensar de manera crítica. En este punto surge la importancia de revisar los procesos de formación docente, dado que un giro pedagógico de la naturaleza que aquí se plantea, involucra grandes retos para el maestro, no solo en su formación pedagógica y disciplinar (ciencia) sino, sobre todo, en el desarrollo de actitudes propicias para fungir como guía y aprendiz en el proceso de aprendizaje de los infantes, más allá del tradicional papel de enseñante.

Como punto de partida, se requiere hacer una revisión crítica de la práctica docente referida a la enseñanza de la ciencia. En este sentido, Cruz-Guzmán et al. (2020) identifican en el diseño de situaciones didácticas sobre ciencia realizadas por educadoras en formación inicial, una enseñanza tradicional reflejo de la escasa libertad que se da al niño de participar en la construcción del conocimiento. En este tipo de enseñanza predominan las actividades dirigidas por el maestro, y se revela una formación disciplinar insuficiente (ciencia). Por lo tanto, para avanzar hacia una pedagogía de preguntas -considerada por Freire y Faundez (2013) como "la única educación creativa y apta para estimular la capacidad humana de asombrarse, de responder a su asombro y resolver sus verdaderos problemas esenciales, existenciales, y el propio conocimiento" (p. 59)- es preciso fortalecer la formación docente. Zuleta (2005) coincide en este necesario giro pedagógico:

Tenemos que cambiar aquellos procesos de enseñanza dogmática, represivos y verticales, por nuevos estilos que sean democráticos, humanistas, participativos, polémicos y críticos, [...] para que las actuales y las futuras generaciones lleguen a ser hombres y mujeres deliberantes con libertad de decisión y elección, y comprometidos con los nuevos valores y con los cambios sociales, económicos y políticos que exige el mundo en que vive (p. 119).

Por el contrario, de persistir un estilo de enseñanza proclive a minimizar la curiosidad como vehículo de aprendizaje y descubrimiento, al aprender a callarse en la escuela, el niño se callará toda la vida (Tonucci, 2008). Entonces, o bien, el niño persiste en su innata disposición a cuestionar y a tomar acción en su forma de aproximarse a la comprensión del mundo o, aprenderá a silenciar su voz y a asumir el conocimiento como verdad absoluta. Si deshacemos esta disyuntiva y afirmamos la primera posibilidad, el papel de los maestros en el aula estará centrado en plantear y escuchar preguntas acerca de los problemas prácticos de la vida de sus alumnos o de sus comunidades (Zuleta, 2005). En otras palabras, este tipo de relación pedagógica demanda mantener, de acuerdo con Zuleta, un diálogo constante donde primen relaciones sociales horizontales que operen como comunidades de aprendizaje (Jiménez, 2012).

A partir de lo anterior, emerge una primera característica o función de la educación científica en los primeros años de escolaridad: potenciar la curiosidad innata mediante la promoción de actitudes investigativas y exploratorias al formular preguntas y plantear hipótesis, las cuales coinciden con las primeras etapas de la indagación científica. Para Torres (2010) este es un rasgo común entre los modelos pedagógicos de las principales tendencias contemporáneas de educación científica (investigación 
dirigida, aprendizaje por descubrimiento, aprendizaje por indagación y el enfoque ciencia, tecnología y sociedad), es decir, la intención de formar pequeños científicos que puedan construir significados sobre lo que les asombra bajo principios de transversalidad e interdisciplinariedad, a través de procedimientos enseñanza-aprendizaje basados en la apertura y el respeto, donde todos los conocimientos e ideas son tan válidos como valiosos. ${ }^{2}$

Sobre la pertinencia de una educación científica situada y crítica, Porlán et al. (1998) identifican cuatro componentes en la enseñanza de la ciencia en educación infantil: la imagen de la ciencia, el modelo didáctico personal, la teoría subjetiva del aprendizaje y el enfoque curricular. La correlación de estos modelos condujo a los investigadores a plantear cuatro niveles de formulación de epistemología escolar: producto formal, proceso técnico, proceso espontáneo y proceso complejo (ver Tabla 1).

Tabla 1. Progresión en epistemología escolar

\begin{tabular}{|c|c|c|c|c|c|c|}
\hline \multirow{2}{*}{$\begin{array}{c}\text { Epistemología } \\
\text { escolar }\end{array}$} & \multirow{2}{*}{$\begin{array}{l}\text { Imagen de } \\
\text { la ciencia }\end{array}$} & \multirow{2}{*}{$\begin{array}{l}\text { Modelo } \\
\text { didáctico } \\
\text { personal }\end{array}$} & \multirow{2}{*}{$\begin{array}{c}\text { Teoría } \\
\text { subjetiva del } \\
\text { aprendizaje }\end{array}$} & \multicolumn{3}{|c|}{ Enfoque curricular } \\
\hline & & & & Contenidos & Metodología & Evaluación \\
\hline $\begin{array}{l}\text { Conocimien- } \\
\text { to escolar } \\
\text { como pro- } \\
\text { ducto formal }\end{array}$ & $\begin{array}{l}\text { Raciona- } \\
\text { lismo }\end{array}$ & Tradicional & $\begin{array}{l}\text { Apropiación } \\
\text { formal de } \\
\text { significados }\end{array}$ & $\begin{array}{l}\text { Reproduc- } \\
\text { ción y sim- } \\
\text { plificación } \\
\text { disciplinar }\end{array}$ & $\begin{array}{l}\text { Transmi- } \\
\text { sión verbal } \\
\text { del profesor }\end{array}$ & $\begin{array}{l}\text { Califica- } \\
\text { ción (exá- } \\
\text { menes) }\end{array}$ \\
\hline $\begin{array}{l}\text { Conocimien- } \\
\text { to escolar } \\
\text { como proce- } \\
\text { so técnico }\end{array}$ & \multirow{2}{*}{ Empirismo } & \multirow{2}{*}{$\begin{array}{l}\text { Tecnológico } \\
\text { Esponta- } \\
\text { neísta }\end{array}$} & \multirow{2}{*}{$\begin{array}{l}\text { Asimilación } \\
\text { de significa- } \\
\text { dos }\end{array}$} & $\begin{array}{l}\text { Adaptación } \\
\text { disciplinar }\end{array}$ & $\begin{array}{l}\text { Secuencia } \\
\text { cerrada de } \\
\text { actividades }\end{array}$ & $\begin{array}{l}\text { Medida del } \\
\text { grado de } \\
\text { consecu- } \\
\text { ción de los } \\
\text { objetivos }\end{array}$ \\
\hline $\begin{array}{l}\text { Conocimien- } \\
\text { to escolar } \\
\text { como proce- } \\
\text { so espontá- } \\
\text { neo }\end{array}$ & & & & $\begin{array}{l}\text { Adaptación } \\
\text { contextual }\end{array}$ & $\begin{array}{l}\text { Secuencia } \\
\text { orientada } \\
\text { por los inte- } \\
\text { reses de los } \\
\text { alumnos }\end{array}$ & $\begin{array}{l}\text { Participa- } \\
\text { ción en la } \\
\text { dinámica } \\
\text { de la clase }\end{array}$ \\
\hline $\begin{array}{l}\text { Conocimien- } \\
\text { to escolar } \\
\text { como proce- } \\
\text { so complejo }\end{array}$ & $\begin{array}{l}\text { Relativis- } \\
\text { mo mode- } \\
\text { rado }\end{array}$ & $\begin{array}{l}\text { Alternativo, } \\
\text { construc- } \\
\text { tivista e } \\
\text { investiga- } \\
\text { tivo }\end{array}$ & $\begin{array}{l}\text { Construc- } \\
\text { ción de } \\
\text { significados }\end{array}$ & $\begin{array}{l}\text { Reelabo- } \\
\text { ración e } \\
\text { integración } \\
\text { de cono- } \\
\text { cimientos } \\
\text { diversos }\end{array}$ & $\begin{array}{l}\text { Investiga- } \\
\text { ción escolar } \\
\text { de proble- } \\
\text { mas signifi- } \\
\text { cativos }\end{array}$ & $\begin{array}{l}\text { Investiga- } \\
\text { ción de la } \\
\text { hipótesis } \\
\text { curricular }\end{array}$ \\
\hline
\end{tabular}

Fuente: Porlán et al. (1998, p. 286).

A partir de esta clasificación, se enfatiza que una educación científica de mayor pertinencia a las características descritas sobre la curiosidad infantil se relaciona estrechamente con las categorías constituyentes de una epistemología escolar donde el conocimiento es visto como proceso complejo, y donde la imagen de la ciencia se propone desde un relativismo moderado sobre el conocimiento. En esta categoría, el docente asume un modelo de enseñanza centrado en la investigación, que subyace en supuestos teóricos constructivistas, dado que el conocimiento se construye a partir de diferentes experiencias. Dicho modelo integra un enfoque curricular abierto y flexible, donde se pueden incluir diversos conocimientos bajo los enfoques de transversalidad

2 Torres (2010) analiza ampliamente las principales tendencias de educación científica. 
e interdisciplinariedad, cuyo punto de partida son los intereses o problemas significativos de los niños. Por último, se observa que la evaluación se centra en el proceso y no en el producto.

Estos rasgos curriculares no son distantes a los planteamientos curriculares de educación preescolar en México, donde el docente a partir del carácter abierto y flexible del currículo, es quien decide las prioridades de aprendizaje y las respectivas rutas metodológicas, en congruencia con las características del contexto socioeducativo (SEP, 2017). Respecto a la evaluación se propone poner atención sobre cómo los niños progresan en sus aprendizajes, así como el reconocimiento de los retos, es decir, una evaluación formativa (SEP, 2017).

La educación preescolar es un potente agente socializador que participa de la significación e interacción en el mundo con una gran responsabilidad sobre las interacciones que promueve, especialmente en contextos de mayor vulnerabilidad en la población (Meece, 2000; Jarillo, 2014). Si partimos del supuesto de que las relaciones con el mundo son las que permiten a los sujetos, a partir de su socialización, definir quiénes son (Berger y Luckmann, 1995), y esto se operacionaliza al asumir roles dentro los grupos sociales donde se construyen patrones de interacción con los otros y con el ambiente (Porras y Pérez, 2019), entonces el potencial de la educación preescolar radica precisamente en ser el primer espacio social de educación formal obligatoria, que contribuye en la formación de las bases sobre las cuales se construirán futuros aprendizajes acerca de los procesos de interacción y significación del ambiente. Esta es la relevancia de las relaciones de aprendizaje en el nivel preescolar para la formación de ciudadanos capaces de establecer relaciones sustentables en lo social y con el ambiente.

Además de los dos niveles mencionados (lo social y el ambiente), Sauvé (1999) menciona un primer nivel de interacción del individuo, ubicado en la esfera de construcción de la identidad: uno mismo (véase Figura 1).

Figura 1. Las tres esferas interrelacionadas del desarrollo personal y social y el ambiente

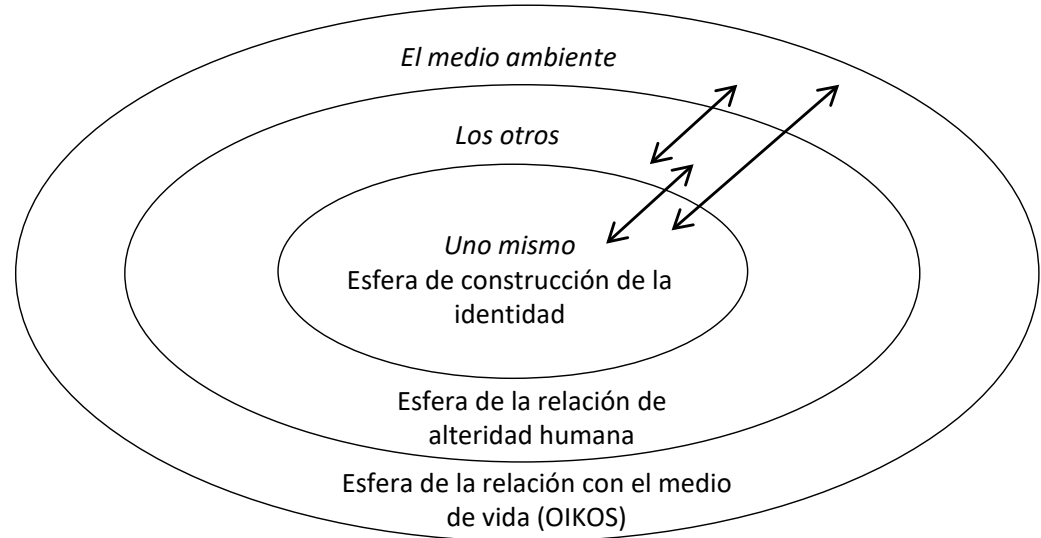

Fuente: Sauvé (1999, p. 15). 
Por ello la importancia de la educación formal de la primera infancia, que media la significación e interacción al inicio de la vida, y en la que el desarrollo del lenguaje, la interacción y las emociones son fundamentales, para la formación de la propia identidad. En este punto de la discusión incorporamos la noción de sustentabilidad como criterio ético que guía las interacciones de desarrollo personal, social y con el ambiente que el sujeto requiere desarrollar desde la infancia y a lo largo de su vida (Ricketts, 2010).

Por lo tanto, la pertinencia de una educación científica con perspectiva ecológica y social para la formación de personas-ciudadanos radica también en el reconocimiento de relaciones sociales bidireccionales, en las que los niños ponen en juego su capacidad de participación, apropiación, reformulación y transformación de lo que reciben mediante la socialización con otros agentes sociales (Pilotti, 2001). Al respecto, Simkin y Becerra (2013) identifican un cambio fundamental en la manera de concebir los procesos de socialización durante el siglo $\mathrm{XX}$, la cual parte de una visión estructuralista de origen, centrada en el papel que ejerce la sociedad sobre el individuo, hacia el reconocimiento de las relaciones sociales bidireccionales y, en éstas, la participación protagónica que ejercen los niños como agentes sociales. Bajo este nuevo paradigma relacional, la socialización se asume como "un proceso de apropiación, reinvención y transformación, en el que ocupa un lugar preferente la actividad grupal" y mediante el cual, los niños participan como co-constructores de su realidad (Rodríguez, 2007, p. 63), donde el niño "es un sujeto complejo capaz de sorprendernos por su autonomía y capacidad reflexiva" (p. 152). Una consecuencia de este cambio cultural es la disposición a reconocer que ellos están listos para hablar, que son actores relevantes de sus sociedades, y que participan activamente en los espacios sociales, reconfigurándolos y transformándolos.

Si ha quedado claro que los niños no son simples receptores en las relaciones sociales que establecen, ha de aceptarse que los infantes no solo construyen significados acerca del ambiente y su relación con este, como ciudadanos futuros, sino como ciudadanos en tiempo presente. Sobre este punto, Lister (2007) propone reconocer cómo los niños son constituidos a través de su manera de vivir su ciudadanía en una relación dialéctica presente y no solo como ciudadanos sujetos de derecho o en proceso de formación. Es decir, que éstos son constituidos e interpelados por lo que están viviendo. Debido a ello, Hayward (2021), afirma que "necesitamos poner mayor atención a las interacciones de los micro y macro niveles de cambio que están tomando lugar en el medio ambiente de los niños" (p. 18), puesto que, cada vez más, los jóvenes e infantes están tomando acciones ante los problemas ambientales, los cuales forman parte de su vida, y están marcando el rumbo de una ciudadanía activa y comprometida con el cambio.

La participación activa que se observa no es fortuita: en los casos donde se revela una agencia social fortalecida, los niños y los jóvenes han sido formados a través de metodologías activas que apuestan al pensamiento crítico y creativo, como el caso de jóvenes de Nueva Zelanda (documentado por Hayward, 2021) y en ciertos estadios sociales alrededor del mundo ${ }^{3}$. ${ }^{3}$ Acerca de la noción de agencia social, Giddens (1986, p. 9) afirma que no se refiere a las intenciones que la gente
tiene en hacer cosas, sino a su capacidad de hacerlas, lo cual implica poder y posibilidad. 
Entonces, si se pretende la construcción de una sociedad justa y sostenible, se deben diseñar y ofrecer experiencias formativas que promuevan este tipo de participación social, donde educadores logren dar voz a sus estudiantes y les ayuden a superar la inmovilidad ante la complejidad y magnitud de la emergencia planetaria, para traducirlo en acciones de compromiso a través de la construcción y la expresión de una ecociudadanía global.

\section{La educación científica y la educación ambiental, un vínculo necesario para enfrentar escenarios socioambientales emergentes}

Como resultado de la discusión previa se sostiene la relevancia de vincular una educación científica situada, crítica y dinámica con una educación ambiental que parta de la noción de complejidad, como nueva racionalidad para significar nuestra relación con el mundo (Leff, 2011). Si bien, el saber ambiental emerge del cuestionamiento a la racionalidad dominante que se asocia al saber científico (Leff, 2004), gracias a la tensión entre estos saberes se ha logrado la transformación de los paradigmas de construcción del conocimiento, dando paso a una mayor aprehensión de la complejidad del mundo. Esto otorga fundamento a la pertinencia de modelos didácticos que integren estrategias interdisciplinarias y contribuyan a una mejor comprensión de la realidad.

También es cierto que, si bien la ciencia y el desarrollo tecnológico han proporcionado innumerables beneficios para la vida humana, a buena parte de ellos se le atribuye el deterioro ambiental (Manifiesto por la vida, 2002), expresado en la pérdida de biodiversidad y el cambio climático, como los mayores desafíos ${ }^{4}$. Pero, más allá de una discusión esencialista sobre la influencia de la ciencia en la crisis planetaria, se intenta exponer la manera cómo las perspectivas hegemónicas de conocimiento y comprensión del mundo (ciencia) instauradas desde la modernidad (en busca de conocimientos objetivos, fragmentados y especializados) han contribuido en el desarrollo de una ciencia que no responde a los escenarios actuales (Leff, 2007; Torres, 2010). En otras palabras, siguiendo a Leff (2007), la crisis ambiental es a la vez una crisis del conocimiento, por lo cual se propone transitar hacia una ciencia que integre dimensiones éticas y políticas para la formación de una ciudadanía crítica:

La cuestión ambiental, más que una problemática ecológica, es una crisis del pensamiento y del entendimiento, de la ontología y de la epistemología con las que la civilización occidental ha comprendido el ser, a los entes y a las cosas; de la racionalidad científica y tecnológica con la que ha sido dominada la naturaleza y economizado el mundo moderno; de las relaciones e interdependencias entre estos procesos materiales y simbólicos, naturales y tecnológicos. (p. 5)

Morín (1999) ha cuestionado la racionalidad que solo sabe separar, la cual ha impulsado la fragmentación del conocimiento y ha atrofiado las facultades de comprensión y de reflexión. El cambio de cosmovisión del mundo y, en consecuencia, de las formas de ser, pensar y actuar, son elementos clave en la ambientalización curricular, más allá de diseñar "cápsulas curriculares" que responden a situaciones didácticas desvinculadas y aisladas (Calafell y Junycent, 2017).

\footnotetext{
${ }^{4} \mathrm{Al}$ respecto véase el reporte 2019 de la Intergovernmental Science-Policy Platform on Biodiversity and Ecosystem Services (IPBES) (en: https://ipbes.net/global-assessment), y los reportes especiales generados por el Panel Intergubernamental de Cambio Climático (IPCC, por sus siglas en inglés) entre 2018 y 2019.
} 
La propuesta de Calafell y Junycent (2017) es integrar a la perspectiva de la complejidad en la práctica docente para cambiar la forma de educar, esto es, educar desde la complejidad para interpretar crítica y creativamente el mundo, y superar las cegueras con las que lo hemos mirado, gracias al pensamiento hegemónico, dicotómico, fragmentado, desarticulado, individualista, racionalista y de dominación. Por el contrario, la educación desde la complejidad aspira un pensamiento divergente, holístico, transdisciplinario, situado, crítico y en colaboración, donde se integran diversas formas de comprender el mundo (Calafell, 2021).

A partir de estas ideas se identifican algunos puntos de coincidencia entre la educación científica y la educación ambiental, como son los enfoques de transversalidad, interdisciplinariedad y complejidad, con base en la integración de saberes conceptuales, procedimentales, actitudinales, axiológicos y políticos, en una propuesta holística de intervención educativa para la construcción de aprendizajes sobre nuestra relación en el ambiente. Estos campos coinciden, además, en las bases pedagógicas de una educación activa, donde el aprendiz es mucho más que un receptor de conocimientos, es decir, toma un papel activo en la construcción de sus conocimientos. FernándezArroyo, Puig-Gutiérrez y García (2017) sugieren apostar "por una educación basada en el aprendizaje significativo, la investigación de problemas, la creatividad, el espíritu crítico, el pensamiento complejo (al modo de Edgar Morín), el conocimiento científico y el trabajo cooperativo, pues de esta manera incrementaremos nuestra resiliencia" (p. 4870).

En síntesis, una educación científica situada, compleja y crítica, parte de los intereses de los educandos, pero como se ha argumentado, el desafío no solo está en escuchar a los infantes, sino guiarlos durante la búsqueda de respuestas, poniendo mayor énfasis en las actitudes, las emociones y las habilidades de pensamiento, a la vez de generar ambientes propicios para acercarlos a diversos entornos, sembrando en ellos el interés de comprenderlos y transformarlos de manera colaborativa, al reconocer su capacidad de acción y compromiso con el entorno. Para Calafell (2021) una metodología activa y colaborativa a partir de preguntas resulta la mejor estrategia para enfrentar tal desafío.

Aunado a lo anterior, las perspectivas contemporáneas de educación científica, la comprensión de su incidencia en los modos de vida social, la apertura de las epistemologías del conocimiento y, en consecuencia, la reubicación de la ciencia sobre la validación de otros saberes ha generado cada vez más el entrecruzamiento de la educación científica con la educación ambiental, definiendo su complementariedad en varios puntos (Sauvé, 2010). Tal como se ha expuesto, si bien el origen de estos campos de conocimiento supondría el antagonismo entre ellos -dado que la ciencia se ha centrado en la comprensión-construcción del mundo como lo conocemos, mientras la educación ambiental emerge como contracorriente, con nueva racionalidad para deconstruir ese mundo (Leff, 2004, 2007)- es la evolución de la educación científica la que ha permitido un nuevo vínculo entre dichos campos educativos. Más aún, la necesaria integración de perspectivas contemporáneas involucra también la integración de diferentes saberes (tan válidos como los científicos), como son los 
saberes de la experiencia, los saberes tradicionales y los saberes del sentido común, que constituyen formas igualmente valiosas para comprender y relacionarnos con el mundo (Sauvé, 2010).

De esta manera, la educación científica contemporánea se abre cada vez más a la necesidad de superar una perspectiva positivista centrada en el desarrollo de habilidades cognitivas, para dar paso a una educación científica más amplia, que integra otros saberes en la construcción del conocimiento, además de nuevas prácticas de enseñanza y aprendizaje en las aulas. Sauvé (2010), al analizar las corrientes de educación científica: ciencia, tecnología, sociedad y ambiente; alfabetización científica y tecnológica; y para la acción política, identifica como un punto de coincidencia a la incorporación de las cuestiones socioecológicas. Entonces, el medio ambiente además de ser el contenido idóneo para el estudio científico de las realidades, es el eje central para el diseño de intervenciones pedagógicas donde prime el trabajo colaborativo, la gestión de proyectos, la investigación y la motivación por seguir aprendiendo (Sauvé, 2010).

Se ha de precisar que, más que un objeto de estudio, el ambiente es "un lugar de vida, el lugar donde vivimos, donde nos transformamos y del cual somos parte, un conjunto de realidades vivas con las cuales entramos en acción" (Sauvé, 2013a, p. 11). Dicho lo anterior, la escuela infantil necesita poner especial atención en la significación que construye sobre el medio ambiente, el cual es el eje central de una educación científica con sentido socioecológico. Por lo tanto, toda acción educativa con infantes tendrá por fuerza que considerar lo anterior si busca generar una verdadera formación-transformación humana en la escala y el sentido existencial requeridos. Se trata entonces de significar al medio ambiente como un lugar de vida y, en consecuencia, un lugar de compromiso, puesto que sin compromiso no hay acción (Sauvé, 2013a).

Aquí cobra relevancia incorporar junto con el pensamiento crítico y creativo al pensamiento cuidante propuesto por Agúndez (2018) en su programa de filosofía para niños, a través del cual "el sujeto no solo es capaz de percibir el mundo, sino de imaginar el mundo en el que le gustaría vivir, de imaginar tanto lo que el mundo podría ser como lo que debería ser" (p. 687). El pensamiento cuidante permite al sujeto moral preocuparse por el otro, condición indispensable en la formación del compromiso para la acción. Este tipo de pensamiento se asienta en bases éticas y estéticas, es decir, en el sentir y en el deber ser, además del pensar, imaginar y crear. Implica, en otras palabras, integrar al conocimiento científico, emociones (estético), principios y valores universales (ético), y la participación-acción (político).

A la luz de estas ideas, la complementariedad entre la educación ambiental y la educación científica es posible gracias a que la educación científica facilita la comprensión del ambiente, mientras la educación ambiental promueve la transformación de las formas de relación en y con el ambiente en busca de modos de vida más justos y sostenibles. En función de esta articulación, Sauvé (2013b) propone una educación eco-científica cuyo objetivo es formar ciudadanos críticos en la comprensión de la realidad, la toma de decisiones y la acción para su transformación. De ahí la necesidad de enfatizar sobre la integración de saberes de tipo ético, estético, crítico y político 
en la educación contemporánea para la formación de una eco-ciudadanía interesada en la toma de decisiones públicas y la evolución de la sociedad de la producción y el consumo en una eco-sociedad.

Cabe advertir que, si bien la educación en ciencia y tecnología permite una aproximación al patrimonio cultural y a la construcción del conocimiento, "por sí sola [...] no es pertinente para otra función de la educación como pueda ser el autodesarrollo de la conciencia de la persona como ciudadana activa, participativa y creativa" (Gutiérrez-Bastida, 2019, p. 108). Es decir, se precisa una educación comprometida con nuevas formas de ser y estar en el mundo, lo cual se alinea a los Objetivos para el Desarrollo Sostenible, que instan a la comunidad internacional a cambiar el estilo de desarrollo dominante, lo que implica un cambio en la relación y concepción del medio ambiente (Naciones Unidas, 2018). Sin embargo, este cambio de paradigma tendrá que ser mucho más ambicioso, pues demanda cuestionar el énfasis en el crecimiento, como el modelo socioeconómico y político hegemónico, que cosifica todo y se centra en la acumulación de capital como garante de la calidad de vida en oposición a otras cosmovisiones que priorizan a la armonía entre las formas de vida como condición indispensable para alcanzar la calidad de vida (Le Grange, 2019; Chuji, Rengifo y Gudynas, 2019).

Así, de la integración de los enfoques eco-científico y eco-social resulta una educación científica, ecológica y social capaz de encender "la indignación ante las expresiones de injusticia social y ecológica y que le oriente hacia la reflexión, la responsabilidad, el compromiso y la acción transformadora a favor de la justicia social y la sostenibilidad de la vida" (Gutiérrez-Bastida, 2019, p. 108). La educación que se propone alude a un enfoque de educación que integra bases éticas y políticas para la formación de una ecociudadanía, para la promoción de nuevas formas de estar en el mundo centrada en "vivir aquí juntos", con responsabilidad colectiva respecto a los sistemas de vida de los que formamos parte (Sauvé, 2014). Para ello, Sauvé (2013a, p. 10) propone "...formar ciudadanos capaces de jugar de manera eficaz y rigurosa su rol de 'lanzadores de alertas' y de participar en la gobernanza ambiental...", lo cual se complementa con el planteamiento de Gutiérrez-Bastida (2019) sobre una educación "implicada en la transformación eco-social que ayuda a revelar una renovada cosmovisión del universo y una ética eco-social donde las personas desarrollan sus capacidades y aptitudes plenamente" (p.111).

Ambas propuestas, eco-científica y eco-social, permiten la convergencia de una formación ética, política y científica para la construcción de mundos distintos, lo cual es posible y necesario, pues estamos en el tiempo del "todavía no" (Herrero, 2016), lo cual refleja potencialidad y esperanza. Además, las dos propuestas tienen la convicción de que la solidaridad urgente es con la generación de seres humanos que sufren sus consecuencias hoy en día, además de las generaciones futuras (Agúndez, 2021).

En síntesis, una educación científica sin una mirada ecológica y social carece de pertinencia, pues no responde a la solución de las problemáticas socioambientales existentes; en todo caso, una educación científica apolítica, neutral, individualista y en exceso racionalista, es parte del problema ambiental que enfrentamos como humanidad. Por lo tanto, la educación científica vinculada a educación ambiental, como lo 
proponen los enfoques eco-científico y eco-social, configuran un solo frente para ayudar a transformar nuestra relación en y con el ambiente, más allá de pensar paliativos para mitigar los efectos del modelo de vida hegemónico insostenible.

\section{Conclusiones}

Con la intención de articular el cierre de estas reflexiones, si bien se reconoce que la discusión sobre la educación científica y ambiental con y para la primera infancia está abierta, desde el análisis expuesto se valora a la educación científica con perspectiva ecológica y social como una alternativa que permita superar el reduccionismo de la educación científica en la práctica educativa con infantes. Esto, con base en las aproximaciones teóricas discutidas, pero también en las aproximaciones empíricas con docentes sensibles y capaces de desarrollar una práctica educativa que recupera los intereses y apegos de los preescolares, en el interés de poner en marcha una educación científica que, por un lado, supere las limitaciones de la tradición positivista arraigada en la ciencia, y al mismo tiempo amplíe las oportunidades de aprendizaje para ejercer su ciudadanía activa. Una formación que permita a los niños sentar las bases para la comprensión de la complejidad ambiental y que, a su vez, contribuya a la transformación de las formas de relación en y con el ambiente, en otras palabras, enseñar ciencia con conciencia (Porlán, 2018).

Lo central con la primera infancia en materia de educación científica y ambiental es recrear, en la inteligencia, la emoción y la relacionalidad ${ }^{5}$; nuevas formas de ser, saber y estar con el ambiente, concebido como un lugar de vida, un lugar de compromiso con la vida y los sistemas de vida. La importancia de una educación científica con estas características desde edad temprana se inscribe en una postura que integra saberes conceptuales, procedimentales, éticos, estéticos y políticos; es decir, no se reduce a procesos de transmisión de conocimientos científicos o habilidades de pensamiento.

Conviene insistir en el riesgo de contribuir a la conservación de relaciones basadas en el dominio al no integrar una postura ética y política en la educación científica acerca de la comprensión del medio ambiente, además de apoyar la formación de visiones fragmentadas y desarticuladas. En otras palabras, se convoca a promover en los jóvenes estudiantes un pensamiento crítico para cuestionar, sobre todo a partir de la emoción, para generar el compromiso con la transformación de la realidad mediante el pensamiento cuidante, creativo y divergente, a partir de la formación de una identidad personal y colectiva de respeto, amor y colaboración, reconociéndonos como seres eco-dependientes e interdependientes.

En este escenario, la educación eco-socio-científica es una alternativa pertinente para reconstruir la red de conexiones de vida de manera justa y sostenible, que integra una unión entre el saber, el sentir, el deber y el actuar, de la cual se nutre el campo de la educación para la transformación (Gutiérrez-Bastida, 2019). En ello subyace la gran responsabilidad para la educación científica en esta era, especialmente con los más

\footnotetext{
${ }^{5}$ La relacionalidad es una noción referida por teóricos de la sociología y la antropología. Alude a la condición epistemológica del pensamiento académico que establece conexiones y relaciones (Mignolo y Walsh, 2018), por una parte, y también a una condición ontológica humana de vinculación con su entorno, presente en las filosofías de culturas prehispánicas de América Latina, y que ha sido estudiada y teorizada por el antropólogo Descola (1986, 2013).
} 
pequeños ciudadanos en estos tiempos. Una educación científica capaz de provocar en la imaginación, el compromiso y el conocimiento a quienes necesitan crecer entre relaciones más justas y sostenibles con el ambiente.

\section{Referencias}

Agúndez, A. (2018). Programa de Filosofía para Niños como propuesta de educación moral: análisis comparado con otros enfoques de la educación moral. Childhood \& Philosophy, 14(31), 671-695. https://doi.org/10.12957/childphilo.2018.34305

Agúndez, A. (2021). Filosofía y educación ecosocial. En Gutiérrez, J. y Quijera, J. (Coords.), 7 saberes para la educación ambiental en la escuela, Módulo 7: cultivar la ética del género humano. Cursos de Verano de la UPV/EHUko Uda Ikastaroak.

Ángel-Maya, A. (2003). La diosa Némesis. Desarrollo sostenible o cambio cultural. Corporación Universitaria Autónoma de Occidente.

Berger, P. y Luckmann, T. (1995). La construcción social de la realidad. Amorrortu Editores.

Buenfil, R. (2006). Los usos de la teoría en investigación educativa. En Jiménez, M. (Coord.), Los usos de la teoría en la investigación (pp. 37-61). Plaza y Valdés.

Calafell, G. (Febrero de 2021). Enfrentar las cegueras del conocimiento: el error y la ilusión. En Gutiérrez-Bastida, J. y Quijera, J. (Coords.), 7 saberes para la educación ambiental en la escuela. Cursos de Verano de la UPV/EHUko Uda lkastaroak.

Calafell, G. y Junycent, M. (2017). La idea vector y sus esferas: una propuesta formativa para la ambientalización curricular desde la complejidad. Teoría educativa, 29(1), 189-216. https:// doi.org/10.14201/teoredu2017291189216

Cañal, P., Travé, G. y Pozuelos, F. (2011). Análisis de obstáculos y dificultades de profesores y estudiantes en la utilización de enfoques de investigación escolar. Investigación en la escuela, 73, 5-26. https://bit.ly/3mxteGJ

Chuji, M., Rengifo, G. y Gudynas, E. (2019). El buen vivir. En Kothari, A., Salleh, A., Escobar, A., Demaria, F. y Acosta, A. (Coords.), Pluriverso Un diccionario del posdesarrollo (pp. 188191). Icaria-Antrazyt.

Cruz-Guzmán, M., Puig, M. y García, A. (2020). ¿Qué tipo de actividades diseñan e implementan en el aula futuros docentes de educación infantil cuando enseñan ciencia mediante rincones de trabajo? Enseñanza de las ciencias, 38(1), 27-45. https://doi.org/10.5565/ rev/ensciencias.2698

Descola, Ph. (1996). Constructing Natures: Symbolic Ecology and Social Practice. In Ph. Descola y G. Pálsson (eds.), Nature and Society. Anthropological Perspectives (pp. 82-102). Routledge.

Descola, Ph. (2013). Beyond Nature and Culture. The University of Chicago Press.

Dewey, J. (1989). Cómo pensamos. Nueva exposición de la relación entre el pensamiento reflexivo y proceso educativo. Paidós.

Fernández-Arroyo, J., Puig-Gutiérrez, M- y García, J. (2017). Menos es más (complejidad). Una reflexión sobre la concepción de complejidad predominante en el pensamiento decrecentista. Enseñanza de las Ciencias: Revista de investigación y experiencias didácticas, 1, $4867-$ 4871. https://bit.ly/3AlOsfx

Ferra, G., Méndez, L. y Castro, R. (2019). Políticas y tendencias en el fomento del pensamiento científico: una visión desde los planes de desarrollo 1998-2018. En B. Morales y B. Colorado (Coords.), La formación inicial docente y la enseñanza de las ciencias: Una investigación evaluativa desde el modelo TPACK (pp. 1-10). Pearson.

Flores, F. (Coord.). (2012). La enseñanza de la ciencia en la educación básica en México. Instituto Nacional para la Evaluación de la Educación. 
Freire, P. y Faundez, A. (2013). Por una pedagogía de la pregunta: crítica a una educación basada en respuestas a preguntas inexistentes. Siglo Veintiuno Editores.

Giddens, A. (1986) The constitution of society. University of California Press.

González-Gaudiano, E. y Meira, P. (2020). Educación para el cambio climático: ¿educar sobre el clima o para el cambio? Perfiles Educativos, 42(168), 157- 174. https://doi.org/10.22201/ iisue.24486167e.2020.168.59464

Gutiérrez-Bastida, J. (2019). Antropoceno: tiempo para la ética ecosocial y la educación ecociudadana. Educación Social, medio ambiente y sostenibilidad, 28, 99-113. https://bit.ly/3iL32Hw

Hayward, B. (2021). Children, Citizenship and Environment. Routledge.

Herrero, Y. (2016). Ecologismo: una cuestión de límites. Encrucijadas. Revista Crítica de Ciencias Sociales, 11(1), 1-22. https://bit.ly/2YxtGg1

Jarillo, B. (2014). Listos para aprender y listos para enseñar. La educación preescolar en México. En L. Santibañez y D. Calderón (Coords.), Los invisibles. Las niñas y los niños de 0 a 6 años: Estado de la Educación en México (pp. 202-224). Mexicanos primero.

Jiménez, M. (2006). Los usos de la teoría en la investigación. Plaza y Valdés.

Jiménez, M. (Coord.). (2012). El aprendizaje de las ciencias: construiry usar herramientas. Colofón/Graó.

Leff, E. (2004). Racionalidad ambiental, otredad y diálogo de saberes. Polis, Revista de la Universidad Bolivariana, 2(7), 1-28. https://bit.ly/3FwbVhV

Leff, E. (2007). La complejidad ambiental. Polis, Revista de la Universidad Bolivariana, 6(16), 1-19. https://bit.ly/3DIr3wF

Leff, E. (2011). Sustentabilidad y racionalidad ambiental: hacia otro programa de sociología ambiental. Revista Mexicana de Sociología, 73(1), 5-46. https://bit.ly/3uR6Lbm

Le Grange, L. (2019). Ubuntu. En A. Kothari, A. Salleh, A. Escobar, F. Demaria y A. Acosta, (Coords.). Pluriverso. Un diccionario del posdesarrollo (pp. 470-473). Icaria-Antrazyt.

Lister, R. (2007). Why Citizenship: Where, When and How Children? Theoretical Inquiries in Law 8(2), 693-717. https://doi.org/10.2202/1565-3404.1165

Manifiesto por la vida. (2002). Manifiesto por la vida. Por una Ética para la Sustentabilidad. Ambiente \& Sociedade,10, 149-162. https://doi.org/10.1590/S1414-753X2002000100012.

Meece, J. (2000). Desarrollo del niño y del adolescente. Secretaría de Educación Pública.

Mignolo, W. y Walsh, C. (2018). Introduction. In Mignolo, W. y Walsh, C. (eds.), On Decoloniality: Concepts, Analytics, Praxis (pp. 1-12). Duke University Press.

Morales, B. y Colorado, B. (Coords.), La formación inicial docente y la enseñanza de las ciencias: Una investigación evaluativa desde el modelo TPACK (pp. 171-183). Pearson.

Morin, E. (1999). Los siete saberes para la educación del futuro. UNESCO.

Naciones Unidas (2018). La Agenda 2030 y los Objetivos de Desarrollo Sostenible: una oportunidad para América Latina y el Caribe. Naciones Unidas.

Ortiz, G. y Cervantes, M. L. (2015). La formación científica en los primeros años de escolaridad. Panorama, 9(17), 10-23. https://doi.org/10.15765/pnrm.v9i17.788

Pilotti, F. (2001). Globalización y convención de los derechos de los niños. El contexto del texto. Naciones Unidas-CEPAL.

Porlán, R. (2018). Didáctica de las ciencias con conciencia. Enseñanza de las ciencias, 36(3), 5-22. https://bit.ly/3akPD40

Porlán, R., Rivero, A. y Martín del Pozo, R. (1997). Conocimiento profesional y epistemología de los profesores I: teoría, métodos e instrumentos. Enseñanza de las Ciencias, 15(2), 155-171. https://bit.ly/2YD2UmL

Porlán, R., Rivero, A. y Martín del Pozo, R. (1998). Conocimiento profesional y epistemología de los profesores II: estudios empíricos y conclusiones. Enseñanza de las Ciencias, 16(2), 271288. https://bit.ly/3uR7dX6 
Porras, Y. y Pérez, M. (2019). Identidad ambiental: múltiples perspectivas. Revista Científica, 34(1), 123-138. https://doi.org/10.14483/23448350.14003

Quintanilla, M., Orellana, C. y Páez, R. (2020). Representaciones epistemológicas sobre competencias de pensamiento científico de educadoras de párvulos en formación. Enseñanza de las Ciencias, 38(1), 47-66. https://bit.ly/3BnlYT6

Rivero, A., Martín del Pozo, R., Solís, E., Azcárate, P. y Porlán, R. (2017). Cambio del conocimiento sobre la enseñanza de las ciencias de futuros maestros. Enseñanza de las Ciencias, 35(1), 29-52. https://bit.ly/3uQVbNw

Ricketts, G. (2010). The Roots of Sustainability. Academic Questions, 23(1), 20-53. https://doi. org/10.1007/s12129-009-9151-5

Rodríguez, P. (2007). Para una sociología de la infancia: aspectos teóricos y metodológicos. Centro de Investigaciones Sociológicas.

Sauvé, L. (1999). La educación ambiental entre la modernidad y la posmodernidad: en busca de un marco educativo de referencia integrador. Tópicos, 1(2), 7-27. https://bit.ly/3lnOgtR

Sauvé, L. (2010). Educación científica y educación ambiental: un cruce fecundo. Enseñanza de las ciencias, 28(1), 5-18. https://bit.ly/3uTSzP0

Sauvé, L. (2013a). Hacia una educación ecocientífica. Revista TED, 34, 7-12. https://doi. org/10.17227/01203916.2107

Sauvé, L. (2013b). Saberes por construir y competencias por desarrollar en la dinámica de los debates socio-ecológicos. Integra educativa, 6(3), 65-87. https://bit.ly/309RaZl

Sauvé, L. (2014). Educación ambiental y ecociudadanía. Dimensiones claves de un proyecto político pedagógico. Revista científica, 18, 12-23. https://bit.ly/3Ao6q0Y

Secretaría de Educación Pública (2011). Programa de Estudio 2011. Guía para la educadora. SEP.

Secretaría de Educación Pública (2017). Aprendizajes clave para la Educación Integral, Educación preescolar, Plan y programas de estudio, orientaciones didácticas y sugerencias de evaluación. SEP.

Simkin, H. y Becerra, G. (2013). El proceso de socialización. Apuntes para su exploración en el campo psicosocial. Ciencia, Docencia y Tecnología, 24(47), 119-142.

Tonucci, F. (1997). La verdadera reforma empieza a los tres años. Revista Investigación en la escuela, 33, 5-16. https://bit.ly/2YD3Ibh

Tonnuci, F. (29 de diciembre de 2008). La misión principal de la escuela ya no es enseñar cosas. La Nación. https://bit.ly/3myVOma

Tonucci, F. (2019). Por qué la infancia: Sobre la necesidad de que nuestras sociedades apuesten definitivamente por las niñas y los niños. Destino.

Torres, M. (2010). La enseñanza tradicional de las ciencias versus las nuevas tendencias educativas. Revista Electrónica Educere, 14(1), 1409-1442. https://bit.ly/3Do57Bm

Zuleta, O. (2005). La pedagogía de la pregunta. Una contribución para el aprendizaje. Educere, 9(28), 115-119. https://bit.ly/3AlkTLb

\section{Cómo citar en APA:}

Martínez-Silva, M. del C., Cruz-Sánchez, G. E. y Aparicio-Cid, R. (2021). La educación científica infantil con perspectiva ecológica y social para la formación de ciudadanías participativas. Revista Iberoamericana de Educación, 87(2), 35-50. https://doi.org/10.35362/rie8724608 